\title{
Renal function change after switching tenofovir disoproxil fumarate for tenofovir alafenamide in the HIV-positive patients of a metropolitan sexual health service
}

\author{
Dane Turner ${ }^{1,2+}$, Douglas Drak ${ }^{1 *}$, Catherine C. O'Connor ${ }^{2,3,4}$, David J. Templeton ${ }^{2,3,4}$ and David M. Gracey ${ }^{2,5}$
}

\begin{abstract}
Background: Tenofovir disoproxil fumarate (TDF) is widely used in the management of HIV-infection, but has been associated with renal impairment in a small proportion of patients. Tenofovir alafenamide (TAF), a novel prodrug of tenofovir, causes less renal impairment and can improve renal function in patients switched from TDF. The factors which predict improved renal function in patients switching from TDF to TAF have yet to be described.
\end{abstract}

Aim: To determine which patient factors are associated with an improvement in renal function following the switch from a TDF- to a TAF-based HIV antiretroviral regimen.

Methods: A retrospective analysis was performed of a cohort from a publicly funded sexual health clinic in Sydney, Australia. All HIV-positive clinic patients switched from a TDF- to TAF-containing regimen between January 2016 and August 2018 were eligible for inclusion. Laboratory results were obtained from patients' electronic medical records. The statistical significance of differences between pre- and post-switch means was determined by paired t-tests, adjusted for baseline values, and associations between continuous variables by univariate linear regression.

Results: 79 patients met inclusion criteria. The majority were male (89\%), with a median age of 44 years (IQR: 34.5 to 53). Patients had a mean pre-switch estimated glomerular filtration rate (eGFR) of $95 \pm 2 \mathrm{~mL} / \mathrm{min} / 1.73 \mathrm{~m}^{2}$, and there was no significant change post-switch $(p=0.062)$. Pre-switch eGFR was a significant predictor of the magnitude of eGFR change after the switch ( $p<0.001)$, but there was no significant association with age $(p=0.189)$, cumulative TDF exposure $(p=0.454)$ or baseline urinary protein to creatinine ratio $(p=0.814)$.

Conclusion: While there was no significant difference in mean eGFR, in patients switched from TDF to TAF, baseline eGFR was a significant predictor of the change in eGFR. This suggests that patients on TDF with poorer baseline renal function would benefit more from switching to TAF. Further study to explore this association is warranted.

Keywords: Tenofovir disoproxil fumarate, Tenofovir alafenamide, HIV, Renal function

\section{Background}

Modern combination antiretroviral therapy (cART) is highly efficacious in virological suppression of HIV-infection [1]. Novel drug development is ongoing however, to improve the tolerability and safety of cART regimens,

*Correspondence: ddra8845@uni.sydney.edu.au

†Dane Turner and Douglas Drak contributed equally to this work

1 Sydney Medical School, University of Sydney, Sydney, NSW, Australia

Full list of author information is available at the end of the article particularly given the need for lifelong therapy. Tenofovir alafenamide (TAF) is a recent product of this initiative and has been shown to be similarly efficacious to the older prodrug tenofovir disoproxil fumarate (TDF) with a lower incidence of adverse renal events [2].

TDF remains widely used and a recommended first-line antiretroviral by major international societies $[1,3]$. It is, however, associated with proximal tubular dysfunction including impaired estimated glomerular filtration rate (eGFR), proteinuria and, rarely, Fanconi syndrome [4]. 
These effects are largely reversible with the discontinuation of TDF $[5,6]$ and recent evidence has emerged that these adverse effects can also be ameliorated by switching to TAF [7-10]. Not all patients benefit equally from the switch from TDF to TAF. One large study of patients with impaired creatinine clearance (30 to $69 \mathrm{~mL} / \mathrm{min}$ ) and multiple comorbidities showed no improvement in eGFR from switching [11].

There are limited other data on factors which may predict improvements in renal function with the switch of antiretroviral regimen. There was no apparent difference in the response of patients with or without pre-existing renal impairment (eGFR $<90 \mathrm{~mL} / \mathrm{min}$ ) in the data supplement of one of the larger switch studies, but no statistical analysis was performed [7]. Our aim was therefore to address this paucity of data and investigate factors, in a clinical setting, which predict improvement in renal function among HIV-positive patients switched from TDF to TAF-containing cART.

\section{Methods}

This study was conducted in accordance with the Declaration of Helsinki and applicable local and national guidelines. Ethics approval for this project was obtained from the RPAH Zone Human Research Ethics Committee of the Sydney Local Health District. This included a waiver of consent, permitting access to identified health data.

A retrospective review was conducted of HIV-positive patients who attend an inner-city publicly funded sexual health service in Sydney, Australia for routine HIV care. Study inclusion criteria were: switching from a TDF- to TAF-containing regimen between January 2016 and August 2018 (inclusive), aged $\geq 18$ years at time of switch and at least one eGFR measurement within the 6 months prior to the switch date and at least one eGFR measurement within 9 months after the switch date. There were no specific exclusion criteria.

HIV medication history and laboratory results were extracted from the electronic medical records of eligible patients. Laboratory results collected were serum creatinine, urinary protein to creatinine ratio (uPCR) and serum phosphate, with eGFR calculated using the CKD-EPI equation [12]. Clinic visits occurred on approximately a 3- to 6-monthly basis and all results were therefore attributed to the nearest 3-month period as measured from the "switch date", defined as the date patients were provided with their first prescription for a TAF-containing regimen. The "pre-switch" measurement was defined as the laboratory result at the switch date or, if unavailable, that immediately preceding it. The "postswitch" measurement was defined as the first laboratory result after at least 3 months post-switch. Both pre- and post-switch measurements were limited to a 6-month window. Where there were multiple measures in a testing period, the mean of test results was calculated and used for the analysis.

Statistical analyses were preformed using SPSS v20 (IBM, New York, USA) and figures were generated using Prism v7 (GraphPad Software, California, USA). Comparisons between pre- and post-switch laboratory measures were performed by using paired $t$-tests with baseline values as a covariate, to adjust for regression to the mean. This procedure increases precision by adjusting for the mean-centered independent variable [13], in our study, the baseline value. Linear regression was used to assess the association of covariates on the change in eGFR post-switch and those with a $\mathrm{p}$-value of $<0.1$ in univariate analysis were considered in the multivariate model. The covariates analysed in univariate analysis were age, cumulative TDF exposure, pre-switch eGFR and preswitch uPCR. Data are presented as either mean \pm SEM or median (IQR). For all analyses, $\mathrm{p}<0.05$ was considered statistically significant.

\section{Results}

A total of 79 HIV-positive patients were identified that had switched from a TDF- to TAF-containing antiretroviral regimen and were included in the analysis. Almost $90 \%$ of patients were male, with a median age of 44 years (IQR 34-53). Patients had been on a TDF-containing regimen for a median of 3.5 (IQR 1.5-4.75) years, with three patients having been on TDF for 3 months or less (Table 1).

Both TDF and TAF were taken by all patients in fixeddose combination tablets, outlined in Table 1. Those patients listed as receiving $\mathrm{TDF} / \mathrm{FTC} /{ }^{\dagger}$ or $\mathrm{TAF} / \mathrm{FTC} /{ }^{\dagger}$ were also on a third agent, not otherwise listed in the table. In over three quarters of patients, both pre and post-switch, this third agent was dolutegravir.

\section{Renal function pre- and post-switch}

All pre- and post-switch measurements occurred 3 to 12 months apart. Prior to switching from their TDFcontaining regimens, patients had an eGFR of $95 \pm 2 \mathrm{~mL} /$ $\mathrm{min} / 1.73 \mathrm{~m}^{2}$, a uPCR of $10.0 \pm 0.9 \mathrm{mg} / \mathrm{mmol}$ and a serum phosphate of $1.05 \pm 0.03 \mathrm{mmol} / \mathrm{L}$. Over one-third of patients (38\%, 95\% CI 27 to 49$)$ had an eGFR $<90 \mathrm{~mL} /$ $\mathrm{min} / 1.73 \mathrm{~m}^{2}$. Proteinuria (uPCR $>20 \mathrm{mg} / \mathrm{mmol}$ ) and hypophosphatemia (serum phosphate $<0.81 \mathrm{mmol} / \mathrm{L}$ ) were present in 4 patients $(5 \%, 95 \%$ CI 0 to $12 \%)$ each, with no instances of patients with both.

Switching to a TAF containing regimen did not lead to significant change in eGFR (mean difference $-2.1 \mathrm{~mL} /$ $\min / 1.73 \mathrm{~m}^{2}, 95 \% \mathrm{CI}-4.3$ to $0.1, \mathrm{p}=0.062$ ) or serum phosphate levels (mean difference $-0.01 \mathrm{mmol} / \mathrm{L}, 95 \%$ 
Table 1 Patient demographics and antiretroviral regimens

\begin{tabular}{ll}
\hline Characteristic & n (\%) or median (IQR) \\
\hline Sex & $70(89)$ \\
Male & $9(11)$ \\
Female & $44(34.5$ to 53) \\
Age (years) & $3.5(1.5$ to 4.75$)$ \\
Cumulative TDF exposure (years) & \\
Pre-switch TDF regimen & $38(48)$ \\
TDF/FTC/ ${ }^{\dagger}$ & $18(23)$ \\
TDF/FTC/RPV & $15(19)$ \\
TDF/FTC/EFV & $8(10)$ \\
TDF/FTC/E/COBI & \\
Post-switch TAF regimen & $57(72)$ \\
TAF/FTC/ ${ }^{\dagger}$ & $12(15)$ \\
TAF/FTC/RPV & $10(13)$ \\
TAF/FTC/E/COBI &
\end{tabular}

$C O B /$ cobicistat, $E$ elvitegravir, EFV efavirenz, eGFR estimated glomerular filtration rate, FTC emtricitabine, $R P V$ rilpivirine, TAF tenofovir alafenamide, TDF tenofovir disoproxil fumarate

† These regimens contained a third agent not otherwise listed in the table. In over three quarters of patients, both pre- and post-switch, the third agent was dolutegravir

$\mathrm{CI}-0.01$ to $0.07, \mathrm{p}=0.818$ ) (Fig. 1 ). Only eight patients had a post-switch uPCR measurement, limiting statistical comparison. The proportions of patients with an eGFR $<90 \mathrm{~mL} / \mathrm{min} / 1.73 \mathrm{~m}^{2}$ (41\%, 95\% CI 34 to $\left.56 \%\right)$ and hypophosphatemia (10\%, 95\% CI 2 to 19$)$ were also similar to pre-switch measures. Although no patients were found to have proteinuria post-switch, uPCR measurements were only available for eight patients.

\section{Predictors of eGFR change}

In a univariate linear model, there was a significant association between pre-switch eGFR and post-switch change in eGFR $(p<0.001)$. There was, however, no significant association between the post-switch change in eGFR and age $(\mathrm{p}=0.189)$, cumulative TDF exposure $(\mathrm{p}=0.454)$ and pre-switch uPCR $(\mathrm{p}=0.814)$ (Table 2$)$. As only a single tested predictor had a $\mathrm{p}<0.1$, no multivariate analysis was performed.

\section{Discussion}

As far as we are aware, this is the first study to explore potential factors which may predict eGFR response in HIV-positive patients switched from TDF to TAF in a real-world setting. We found that, while there was no overall change in eGFR, patients with lower baseline eGFR have a greater improvement in eGFR post-switch.

Of the large randomized controlled studies which have reported on the renal function in patients switching from TDF to TAF-containing regimens, only Gallant

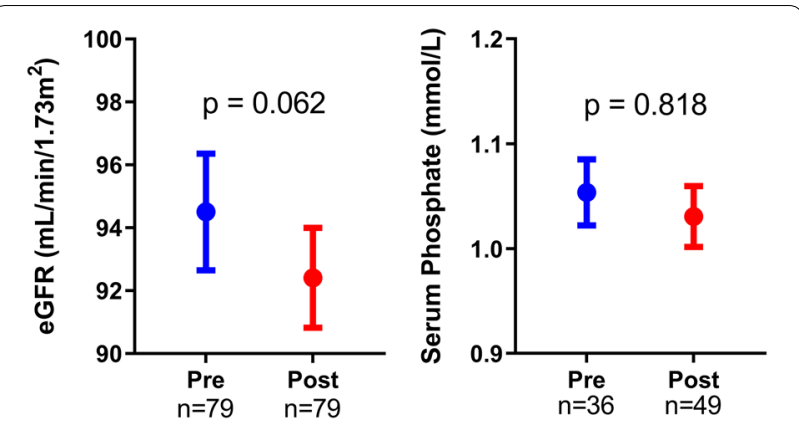

Fig. 1 Measures of renal function in patients before switching from tenofovir disoproxil fumarate (Pre) and after switching to tenofovir alafenamide (Post). Graphs show mean and SEM. eGFR estimated glomerular filtration rate

et al. [7] provided data regarding a differential response in subgroups of the participants. In contrast to our findings, participants with a baseline eGFR $\geq 90$ and those $<90 \mathrm{~mL} / \mathrm{min}$ had a similar eGFR response to switching from TDF to TAF. These same subgroups though had a substantial difference in uPCR. Patients with eGFR $\geq 90$ who remained on TDF showed an increase in UPCR of $5 \%$, whereas those switched to TAF had a decrease of $11 \%$. In contrast, patients with a baseline eGFR $<90$ had a UPCR increase of $16 \%$ on TDF and those on TAF a decrease of $20 \%$. Due to only 3 of our patient group having both pre- and post-switch uPCR results, we were unable to assess this change in our study.

Across the major switch studies, switching from TDF to TAF has had a very robust impact on $\mathrm{UPCR}$ and a comparatively modest effect on eGFR [7-10]. This difference in effect size may account for the apparent disparity between the eGFR and uPCR responses, by baseline eGFR subgroup, reported by Gallant et al. [7]. Furthermore, as renal function was a secondary endpoint in that study and no detailed subgroup analysis was performed, the impact of confounding factors cannot be excluded. As such, our finding of a predictive role of baseline eGFR in determining eGFR response to switching TDF to TAF does not necessarily conflict with the larger Gallant et al. [7] study.

The majority of patients in our study cohort were on a regimen containing one of dolutegravir, cobicistat or rilpivirine, all of which have been shown to inhibit creatinine secretion from the proximal renal tubule, without affecting glomerular filtration [14]. While our sample size did not permit an exploration of the effect of other agents in the cART regimens, this has been demonstrated in other switch-studies. Mills et al. [8], for example, showed that patients switched from TDF/emtricitabine/efavirenz to a TAF regimen which included cobicistat experienced an increase in serum creatine. These effects are 
Table 2 Univariate regression of potential predictors of the change in eGFR in patients switched from TDF to TAF

\begin{tabular}{lcr}
\hline Variable & Coefficient $\mathbf{( 9 5 \% ~ C l )}$ & p value \\
\hline Age & $0.120(-0.080$ to 0.396$)$ & 0.189 \\
Cumulative TDF exposure & $0.025(-0.069$ to 0.031$)$ & 0.454 \\
Pre-switch eGFR & $-0.381(-0.516$ to -0.247$)$ & $<0.001$ \\
Pre-switch uPCR & $0.085(-0.194$ to 0.153$)$ & 0.814 \\
\hline
\end{tabular}

Statistically significant $\mathrm{p}$ value is in italic

eGFR estimated glomerular filtration rate, TAF tenofovir alafenamide, TDF tenofovir disoproxil fumarate

particularly important to consider in real-world clinical settings, where there is likely to be significantly more heterogeneity in cART regimens than clinical trials. It may also account for the trend towards a lower eGFR in postswitch patients in our study, which approached but did not meet statistical significance.

Duration of TDF exposure was not found to predict eGFR change in univariate analysis. Although ongoing TDF exposure has previously been shown to reduce eGFR, the effect is modest [15]. No significant increase in adverse renal events has been observed in patients exposed to TDF for up to 6 years [16]. Given that the majority of our patients had cumulative TDF exposure under 5 years, the lack of association between cumulative TDF exposure and the eGFR response to switching is unsurprising.

Our findings support the recommendation of the European society guidelines, that patients receiving TDF with an eGFR $>60 \mathrm{~mL} / \mathrm{min}$ should be considered for switch if their eGFR has declined by $5 \mathrm{~mL} /$ year for three or more years and/or their eGFR has decreased $>25 \%$ from baseline [1]. This advice comes from expert opinion, pending clinical data. While we recognize the potential for regression to the mean to have influenced our analyses, our observational data suggest that such patients would experience a significant improvement in their eGFR when switched to TAF. It is not reasonable, however, to extrapolate this trend to patients with significant renal impairment $\left(\right.$ eGFR $\left.\leq 60 \mathrm{~mL} / \mathrm{min} / 1.73 \mathrm{~m}^{2}\right)$. No such patients were included in this study, as they would not have been prescribed TDF due to renal concerns. A single study, conducted in this group, found no improvement in eGFR upon switching from TDF to TAF. Of note, participants in this cohort study had a high burden of comorbidities contributing to renal impairment, to which the study authors attributed the lack of response [11]. Available evidence for patients with significant renal impairment, but without significant comorbidities, is presently limited to case reports. These suggest though that the switch to TAF is capable of ameliorating significant TDF-induced renal dysfunction $[17,18]$, even to the point of reversing Fanconi syndrome [19].

The main limitation of this study was the sample size. Although a highly significant trend between lower preswitch eGFR and eGFR improvement post-switch to TAF was observed, further study will be needed in larger patient groups to better characterize the relationship between baseline eGFR change and the eGFR response in patients switched from TDF to TAF-containing regimens. There were also no data collected on comorbidities and renal risk factors. These will be the subject of further investigation, but are unlikely to have compromised the validity of our current findings as these have not been found to be associated with TDF-mediated decline in renal function [20]. Finally, improvements in eGFR and proteinuria have been demonstrated in as little as 4 weeks [10] and these changes have been shown to be durable, lasting until at least 96 weeks after switching [21]. As such, the small variability in the time between pre and post-switch laboratory measurements in our study (between 3 and 12 months) is unlikely to have introduced any significant bias to our findings.

TDF remains a recommended first-line antiretroviral agent in major international guidelines [1,3]. For those patients who go on to experience TDF-induced renal dysfunction, our study suggests that HIV-positive patients with poorer renal function can expect a greater improvement in eGFR upon switching from a TDF- to a TAFcontaining antiretroviral regimen.

\section{Conclusion}

Switching HIV-positive patients from TDF to TAF did not lead to an overall change in eGFR. While there was no significant association between age, cumulative TDF exposure and baseline uPCR in such patients, baseline eGFR was a predictor of eGFR change. This suggests that patients with a lower baseline eGFR on TDF would benefit more from the switch to a TAF-containing regimen, but confirmation awaits larger studies.

\section{Abbreviations}

CART: combination antiretroviral therapy; COBl: cobicistat; E: elvitegravir; EFV: efavirenz; eGFR: estimated glomerular filtration rate; FTC: emtricitabine; RPV: rilpivirine; TAF: tenofovir alafenamide; TDF: tenofovir disoproxil fumarate; UPCR: urinary protein to creatinine ratio.

\section{Acknowledgements \\ Dr Valerie Gracey_for kindly lending her statistical expertise.}

\section{Authors' contributions}

DT was involved in the project design, obtaining ethics approval, data collection/analysis/interpretation and drafting of the manuscript. DD contributed to data collection/analysis/interpretation and drafting of the manuscript. CO, DJT and DMG all contributed to the project design, data interpretation and participated in editing the manuscript. All authors read and approved the final manuscript. 


\section{Funding}

Not applicable.

\section{Availability of data and materials}

The data for our project is retained on the REDCap data system, administered by the Sydney Local Health District. Data availability must be on an "upon reasonable request" basis (made to the corresponding author), to comply with the requirements of the approving human research ethics committee.

\section{Ethics approval and consent to participate}

This study was conducted in accordance with the Declaration of Helsinki and applicable local and national guidelines. Ethics approval for this project was obtained from the RPAH Zone Human Research Ethics Committee of the Sydney Local Health District (Protocol Number X18-0015). This included a waiver of consent, permitting access to identified health data.

\section{Consent for publication}

Not applicable.

\section{Competing interests}

The authors declare that they have no competing interests.

\section{Author details}

1 Sydney Medical School, University of Sydney, Sydney, NSW, Australia. ${ }^{2}$ Central Clinical School, University of Sydney, Sydney, NSW, Australia. ${ }^{3}$ RPA Sexual Health, Sydney Local Health District, Sydney, NSW, Australia. ${ }^{4}$ Kirby Institute, UNSW Sydney, Sydney, NSW, Australia. ${ }^{5}$ Renal Unit, Royal Prince Alfred Hospital, Sydney Local Health District, Sydney, NSW, Australia.

Received: 30 March 2019 Accepted: 28 November 2019

Published online: 07 December 2019

\section{References}

1. (EACS) EACS. European AIDS Clinical Society (EACS) GuidelinesVersion 9.1: European AIDS Clinical Society (EACS); 2018. http://www.eacsociety.org/ files/2018_guidelines-9.1-english.pdf. Accessed 13 Jan 2019.

2. Hill A, Hughes SL, Gotham D, Pozniak AL. Tenofovir alafenamide versus tenofovir disoproxil fumarate: is there a true difference in efficacy and safety? J Virus Erad. 2018;4(2):72-9.

3. Panel on Atriretroviral Guidelines for Adults and Adolescents. Guidelines for the use of antiretroviral agents in adults and adolescents living with HIV. updated Oct 25, 2018. https://aidsinfo.nih.gov/contentfiles/lvgui delines/AdultandAdolescentGL.pdf. Accessed 13 Jan 2019.

4. Hall AM, Hendry BM, Nitsch D, Connolly JO. Tenofovir-associated kidney toxicity in HIV-infected patients: a review of the evidence. Am J Kidney Dis. 2011;57(5):773-80.

5. Mugwanya KK, Wyatt C, Celum C, Donnell D, Kiarie J, Ronald A, et al. Reversibility of glomerular renal function decline in HIV-uninfected men and women discontinuing emtricitabine-tenofovir disoproxi fumarate pre-exposure prophylaxis. J Acquir Immune Def Syndr (1999). 2016;71(4):374-80.

6. Bonjoch A, Echeverria P, Perez-Alvarez N, Puig J, Estany C, Clotet B, et al. High rate of reversibility of renal damage in a cohort of HIV-infected patients receiving tenofovir-containing antiretroviral therapy. Antiviral Res. 2012;96(1):65-9.

7. Gallant JE, Daar ES, Raffi F, Brinson C, Ruane P, DeJesus E, et al. Efficacy and safety of tenofovir alafenamide versus tenofovir disoproxil fumarate given as fixed-dose combinations containing emtricitabine as backbones for treatment of HIV-1 infection in virologically suppressed adults: a randomised, double-blind, active-controlled phase 3 trial. Lancet HIV. 2016;3(4):e158-65.
8. Mills A, Arribas JR, Andrade-Villanueva J, DiPerri G, Van Lunzen J, Koenig $E$, et al. Switching from tenofovir disoproxil fumarate to tenofovir alafenamide in antiretroviral regimens for virologically suppressed adults with HIV-1 infection: a randomised, active-controlled, multicentre, open-label, phase 3, non-inferiority study. Lancet Infect Dis. 2016;16(1):43-52.

9. Orkin C, DeJesus E, Ramgopal M, Crofoot G, Ruane P, LaMarca A, et al. Switching from tenofovir disoproxil fumarate to tenofovir alafenamide coformulated with rilpivirine and emtricitabine in virally suppressed adults with HIV-1 infection: a randomised, double-blind, multicentre, phase 3b, non-inferiority study. Lancet HIV. 2017;4(5):e195-204.

10. Orkin C, Molina JM, Negredo E, Arribas JR, Gathe J, Eron JJ, et al. Efficacy and safety of switching from boosted protease inhibitors plus emtricitabine and tenofovir disoproxil fumarate regimens to single-tablet darunavir, cobicistat, emtricitabine, and tenofovir alafenamide at 48 weeks in adults with virologically suppressed HIV-1 (EMERALD): a phase 3, randomised, non-inferiority trial. Lancet HIV. 2018;5(1):e23-34.

11. Post FA, Tebas P, Clarke A, Cotte L, Short WR, Abram ME, et al. Brief report: switching to tenofovir alafenamide, coformulated with elvitegravir, cobicistat, and emtricitabine, in HIV-infected adults with renal impairment: 96-week results from a single-arm, multicenter, open-label phase 3 study. J Acquir Immune Def Syndr (1999). 2017;74(2):180-4.

12. Levey AS, Stevens LA, Schmid CH, Zhang YL, Castro AF 3rd, Feldman HI, et al. A new equation to estimate glomerular filtration rate. Ann Intern Med. 2009;150(9):604-12.

13. Hedberg EC, Ayers S. The power of a paired t-test with a covariate. Soc Sci Res. 2015;50:277-91.

14. Galizzi N, Galli L, Poli A, Spagnuolo V, Castagna A, Gianotti N. Glomerular filtration rate estimated by cystatin C formulas in HIV-1 patients treated with dolutegravir, rilpivirine or cobicistat. N Microbiol. 2018;41(4):256-61.

15. Martin M, Vanichseni S, Suntharasamai P, Sangkum U, Mock PA, Gvetadze RJ, et al. Renal function of participants in the Bangkok tenofovir studyThailand, 2005-2012. Clin Infect Dis. 2014;59(5):716-24.

16. Mocroft A, Lundgren JD, Ross M, Fux CA, Reiss P, Moranne O, et al. Cumulative and current exposure to potentially nephrotoxic antiretrovirals and development of chronic kidney disease in HIV-positive individuals with a normal baseline estimated glomerular filtration rate: a prospective international cohort study. Lancet HIV. 2016;3(1):e23-32.

17. Gallagher A, Quan D, Gracey DM. Improvement in renal function and resolution of proteinuria in an HIV-infected patient switched from tenofovir disoproxil fumarate to tenofovir alafenamide. Intern Med J. 2017;47(7):826-7.

18. Mikula JM, Manion MM, Maldarelli F, Suarez LM, Norman-Wheeler JF, Ober AG, et al. Tenofovir alafenamide as part of a salvage regimen in a patient with multi-drug resistant HIV and tenofovir-DF-associated renal tubulopathy. Antiviral Ther. 2016;21(6):553-8.

19. Mothobi NZ, Masters J, Marriott DJ. Fanconi syndrome due to tenofovir disoproxil fumarate reversed by switching to tenofovir alafenamide fumarate in an HIV-infected patient. Ther Adv Infect Dis. 2018;5(5):91-5.

20. Scherzer R, Estrella M, Li Y, Choi Al, Deeks SG, Grunfeld C, et al. Association of tenofovir exposure with kidney disease risk in HIV infection. AIDS (London, England). 2012;26(7):867-75.

21. Hagins D, Orkin C, Daar ES, Mills A, Brinson C, DeJesus E, et al. Switching to coformulated rilpivirine (RPV), emtricitabine (FTC) and tenofovir alafenamide from either RPV, FTC and tenofovir disoproxil fumarate (TDF) or efavirenz, FTC and TDF: 96-week results from two randomized clinical trials. HIV Med. 2018;19(10):724-33.

\section{Publisher's Note}

Springer Nature remains neutral with regard to jurisdictional claims in published maps and institutional affiliations. 